\title{
Zweiter Beitrag zur Histogenese des Auges von
}

\author{
Dr. C. Ritter aus Worpswede.
}

Durch das Spiel des Zufalls erhielt ich kurz nach dem ersten einen zweiten noch jüngern menschlichen Embryo. Die Frau war vor nicht ganz zehn Wochen zum letzten Male menstruirt, der Blutfluss hatte acht Tage gedauert. Danach konnte das Ei etwa im Anfang der neunten oder der fünften Woche sein.

Das Fi war etwa $28 \mathrm{Mm}$. gross. Die Decidua umgab es überall und diesen lag der Sack des Chorions an. Nach dem Chorion hin liessen sich die Umbilicalgefässe schon verfolgen. Der Embryo war ungestreckt $5 \mathrm{Mm}$, gestreckt $9 \mathrm{Mm}$. lang. Die Halskrümmung war sehr stark, die vordere etwas geringer. Das Ohr war eben zu bemerken, vom Herzen gingen zwei Kiemenarterien nach jeder Seite ab. Mund- und Nasenhöhle bildeten noch ein Ganzes. Die unteren Extremitäten waren als keine Stummel angedeutet. Die Bauchhöhle war weit offen. Das Amnios umgab den Embryo dicht. Ich hoffe, diese Andeutungen werden genügen, um das Alter des Embryo auf den Anfang der fünften Woche etwa zu bestimmen; eine genauere Untersuchung, welche Professor Meissner in Göttingen die Güte hatte vorzunchmen, hat zu dem- 
selben Resultate geführt, wie meine eigene Untersuchung.

Die Forschung über das erste Entstehen der Gewebe ist mit ausserordentlichen Schwierigkeiten verbunden. Der grossen Seltenheit des Materiales liesse sich durch Brutmaschinen abhelfen, aber unsere Versuchsthiere eignen sich selbst im ausgewachsenen Zustande nicht gut zur Untersuchung der Retina. In jedem einzelnen Falle ist das Material so geringfügig, dass es der Lupe bedarf, um den Bulbus bestimmt erkennen zu lassen. Die geringste ungeschickte Präparation lässt den kleinen Augenpunkt verschwinden und dann ist er nicht sicher wieder aufzufinden. Je mehr der Bulbus seinem ersten Ursprunge nahe steht, um so schwieriger sind die Differenzen der Gewebe zu erkennen; es bedarf schon einer sehr genauen Kunde der histologischen Verhältnisse, um zu einem irgend genügenden Resultate zu gelangen. Auch dem gewandtesten und aufmerksamsten Untersucher wird es nicht möglich werden, alle fraglichen Punkte in einem Präparate zu erledigen; nur sehr genaue Zeichnungen können dann zu längerer Ueberlegung verhelfen. Obgleich ich schon manches schwierige Object untersucht habe, will ich doch offen gestehen, dass es für mich kein angreifenderes Studium giebt, als die Histogenese des Auges. Das Zeichnen unter der Lupe, die vielfachen Combinationen, welche nachzuweisen sind, die Berücksichtigung aller möglichen Verhältnisse machen diese Untersuchungen nicht allein mühevoll, sondern so angreifend, dass ich es wohl verstehe, wie bis jetzt noch keine Entwickelungsgeschichte des Auges mit Berücksichtigung der Histogenese geschrieben ist.

Der einzelne Fall, wie er diesem Aufsatze zu Grunde liegt, soll nur Material herbeischaffen,.die Lösung der zu stellenden Fragen anbahnen; der Grad der Bürgschaft, welche ein einzelner Fall für die gewonnenen Resultate 
darbietet, kann nicht als ein entscheidender angesehen werden. Dennoch halte ich die Veröffentlichung für geboten, weil ein menschlicher Embryo dieses Alters überhaupt nur selten gefunden wird und weil der glückliche Finder nicht immer die genügende Kenntniss und das hinreichende Interesse für die Untersuchung des Auges hat. Da dies der zweite Fall ist, welchen ich untersucht habe, erhöht sich die Sicherheit allerdings bedeutend; allein in dem ersten Falle (dieses Archivs B. X. Abth. 1.) waren alle Gewebe fertig angelegt, während dieser zweite Fall so weit in der Entwickelung zurückliegt, dass sich die Vermittelung zwischen beiden nur durch nicht völlig sichere Schlüsse herstellen lässt.

In diesen Untersuchungen sind drei Punkte zu beachten: 1) die Form der gefundenen Flemente und ihre Verbindungen; 2) die Andeutung auf ihre spätere bleibende Gestalt; 3) die Art und Weise, in welcher sich die Vermehrung der Theile andeutet und die spätere Grösse der Membranen erreicht wird. Es frägt sich, wie weit diese drei Punkte in solchen vereinzelten Beobachtungen erledigt werden können.

Das Auge des menschlichen Embryo aus der fünften Woche ist nur vermittelst der Lupe sicher zu entdecken. Man bemerkt an der vorderen Hirnblase dicht über dem vorderen Ende derselben, also ziemlich weit von der vordersten Kopfkrümmung entfernt, zwei sehr kleine röthliche Punkte. Sie liegen, etwa drei Millimeter von einander entfernt, dicht neben der Mittellinie, zwischen und etwas über ihnen fand sich noch ein kleiner rother Fleck, dessen Deutung ich aber nicht weiter verfolgt habe. Die beiden seitlichen rothen Punkte, die Anlagen der Augen sind über die sonstige Oberfläche des Embryo nicht erhaben; ihre Begrenzung ist weder nach den Seiten, noch nach hinten irgendwie bestimmt; kurzum, obgleich die Anlage der Augen schon ihren bestimmten ort hat, geht 
doch die Form überall in die begrenzenden Theile über oder vielmehr eine geschlossene Form des Bulbus hat sich noch nicht gebildet. Dagegen musste aus dem Lupenbefunde schon vermuthet werden, dass eine gewisse Differencirung der Gewebe bereits begonnen hatte, da die Pigmentirung, wenn auch erst im schwachen Anfange, sich doch deutlich $\mathrm{zu}$ erkennen gab. Jedes Auge bot bei 300 facher Vergrösserung ein Object dar, welches wenig mehr als die Hälfte des Sehfeldes einnahm. Das Bild, welches Ammon in seiner Entwickelungsgeschichte des Auges (dieses Archiv IX. 1.) darstellt ('Tab. I. Fig. 6), muss übrigens bedeutend stärker als vierfach vergrössert sein oder es stellt keinen vierwöchentlichen Embryo dar.

Der Bulbus bestand aus der Hirnblase und der Einstülpung der Oberhaut, welche die Linse bildet. Diese Einstülpung und ihr Stiel schienen mir nicht hohl, sondern solide zu sein, wie dies Ammon schon angegeben hat, obgleich der Versuch mit dem Pferdehaar für ein solches Präparat etwas zu roh gewesen wäre. Der schmale Stiel der Linse bot gewiss kein Lumen dar, dagegen schien die Linse selbst in ihrem Inneren eine kleine Höhlung zu enthalten. Vom Glaskörper habe ich nichts bemerkt, will aber damit nicht behaupten, dass er nicht vorhanden gewesen wäre, nur befand sich seine Anlage sicher nicht zwischen Linse und Retina, welche vielmehr dicht an einander lagen. Die Einstülpung der Retina war schon erfolgt; sie bestand also aus den beiden Blättern der secundären Augenblase, welche aber nicht mehr durch eine Höhlung von einander getrennt waren, wie dies Kölliker gezeichnet hat (Entwickelungsgeschichte des Menschen $u$. d. höheren Thiere Fig. 141). BeideBlätter waren zu einer Membran vereinigt, aber diese noch nicht in späterer Weise ausgespannt, sondern sie erfüllte mehr einen halbkugeligen Raum. Die Bildung der Sclerotica 
und ihrer Adnexa war noch nicht geschehen, das Bildungsmaterial derselben umgab natürlich die Augenblase schon von hinten und seitlich, aber die Differencirung desselben von den Kopfplatten war noch nicht eingetreten, ein eigentlicher Bulbus noch nicht gebildet.

Die Zellen, welche die Kopfplatten und also auch Sclerotica, Chorioidea, Cornea zusammensetzen in dieser Zeit der Entwickelung, stimmen genau mit denjenigen übèrein, aus welchen überhaupt das Bindegewebe, Knorpel und Knochen hervorgeht (Fig. 1). Die Zellenform ist sehr wenig ausgesprochen, ihr äusserer Umriss tritt nur sehr schwach hervor, vielleicht ist eine bestimmte Membran noch nicht gebildet. Die Zelle dehnt sich von einer oder beiden schmalen Seiten des Kernes aus. Der Kern ist gross, länglich rund und enthält einen oder zwei runde Kernkörperchen, sein Längendurchmesser beträgt etwa 0,008 Mm., der Breitendurchmesser 0,005 Mm. Die Form der Kerne zeigte durchaus keine Varianten, auch Theilungsvorgänge liessen sich trotz längeren Suchens nicht entdecken. Bei oberflächlicher Betrachtung schien eine gleichmässige Grundmasse von geringer Ausdehnung die Kerne zu umgeben, erst eine genauere Präparation führte zur Isolation der Zellen, indem ein blasses, kleines Anhängsel an einer Seite des Kernes ihren Umfang zu erkennen gab.

Die Einstülpung der Linse war genau nach der Beschreibung von Huschke vor sich gegangen. Ein grober rundlicher Körper lag hinter der vorderen Augenwand, durch einen Trichter an ihr befestigt. Trichter und Anlagen der Linse bestanden in gleicher Weise aus Zellen, welche denen der Kopfplatten sehr glichen, aber in ihrer Ausbildung wohl etwas weiter vorgeschritten waren (Fig. 2). Die Zellen waren deutlich begrenzt. länglich ausgedehnt, von 0,01 Mm. Länge und 0,004 Mm. Breite. Der grosse Kern hatte einen Durchmesser von 
$0,004 \mathrm{Mm}$. glänzte stark und enthielt regelmässig kein Kernkörperchen. Seine Contour ist gegenüber der Zel. lencontour sehr scharf. Von einer Glasmembran der Kapsel habe ich nichts bemerkt, sie ist in dieser Zeit der Entwickelung noch nicht gebildet.

Die Retina zeigte schon durch ihre bei weitem am bedeutensten fortgeschrittene Entwickelung, dass sie dem Hirne angehört. Es ist nöthig, zur Erklärung der Retinastructur die gleichzeitige Zusammensetzung des Hirns kurz zu erwähnen, um so mehr, alș die Beschreibungen in allen Bücheïn über Entwickelungsgeschichte an diesen Punkten grosse Lücken lassen. Das Gehirn besteht in diesem Zeitmoment der Entwickelung aus deutlich ausgebildeten Zellen mit ziemlich blasser Contour (Fig. 3). Die Form der Zellen ist meist eckig, leicht abgerundet; der Durchmesser beträgt etwa $0,012 \mathrm{Mm}$. Die Kerne sind sehr gross, rund, leicht granulirt, ihr Durchmesser beträgt $0,008 \mathrm{Mm}$. Kernkörperchen habe ich niemals innerhalb der Kerne bemerkt. Die eckige Form der isolirten Zellen findet ihren Grund in den Fortsätzen der Zellen, welche bei genauer Einstellung zu erkennen sind. Meist gehen von der Zelle nach zwei entgegengesetzten Seiten zwei blasse feine Fortsätze ab, deren Verlauf natürlich nicht weiter verfolgt werden kann. Die Ausbildung der Zellen und selbst der Zusammenhang des Gewebes ist also auf dieser Entwickelungsstufe im Gehirne schon fertig und vollendet, während die übrigen Gewebe des Körpers noch aus einer rohen Masse von Zellenund Kernhaufen bestehen.

Die Retina stand mit dem Vorderhirne noch in unmittelbarer Verbindung, ein Sehnerv existirte noch nicht. Durch das Herantreten der Kopfplatten, aus welchen später Sclerotica, Orbita, Muskeln und Fett der Orbita hervorgehen, war die Retina schon eingechlossen und bildete eine kleine Hervorstülpung des Vorderhirns. 
Man hat diese Hervorstülpung als Blase aufgefasst und spricht von zwei Blättern der secundären Blase, wenn dieselbe durch die Bildung der Linse wieder in sich zurückgeschoben wird. Ich habe einen hohlen Raum nicht gefunden, beide Blätter lagen schon unmittelbar an einander und standen in fester Verbindung.

Schon in der früheren Arbeit habe ich mich der Ansicht von Kölliker angeschlossen, dass aus dem äusseren Blatte der secundären Augenblase das Pigmentepithel der Chorioidea und die Stäbchenschicht der Retina hervorgehen, die übrigen Schichten der Retina aber aus dem inneren Blatte. Auch diese vorliegende Beobachtung wird neue Beweise für diese Ansicht herbeischaffen und sie im Einzelnen näher erläutern. In der Masse der Retina liessen sich vier rerschiedene Formen von Zellen unterscheiden, welche in manchen Beziehungen sehr übereinstimmen, durch wesentliche Eigenschaften aber prägnant von einander unterschieden werden kounten. Mochte ihr erster Anfạng gleich gewesen sein, jetzt war schon hinreichende Differenz da, unn ihre spätere Bestimmung festzustellen.

Die erste Form der Zellen (Fig. 4) lag immer in kleinen Haufen von Zellen bei einander. Die richtige Anschauung der Zellen lässt sich nur gewinnen, wenn man die Figur 4 mit der Lupe betrachtet. Die Zellen waren sehr klein, ca. 0,006 Mm. im Durchmesser, illre Contour so wenig scharf und bestimmt, dass sie oft nur mit der grössten Mühe zu finden war. Nicht selten hatte sich von der Begrenzung des Kerns noch gar nicht deutlich eine Zellmembran abgehoben, nur durch wechselnde Einstellung liess sich dann eine zweite sehr blasse Contour um den.Kern herum erkennen. Der runde Kern dagegen lenkte mit Gewalt die Aufmerksamkeit des Beobachters auf sich, er hatte einen Durchmesser von $0,005 \mathrm{Mm}$., glänzte ausserordentlich und war von einer 
scharfen dunklen Contour begrenzt, auf welcher äusserlich kleine schwarze Pünktchen aufhingen. Innerhalb des Kerns lag regelmässig ein scharf begrenztes, gelbrothes Kernkörperchen, der sonstige Inhalt des Kerns war niemals granulirt und glänzte daher so stark, aber zuweilen hafteten dem Kern aussen kleine schwarze Punkte an. Ohne Zweifel ist dies die früheste erkennbare Form der Pigmentepithelien der Chorioidea; die Zellen sind erst eben gebildet, indem die Kerne ihre Function, die Secretion des Pigmentes, begonnen haben. Meine frühere Beobachtung über die Entstehung desselben findet in diesem Falle ihre völlige Bestätigung und ihre Frgänzung bis zum ersten Beginne. Die Pigmentbildung geschieht durch Krystallisation eines innerhalb des Kerns gelösten Farbstoffes auf der äusseren Begrenzung des Kerns. In dem vorliegenden Falle hat dieser Prozess eben erst begonnen und es finden sich daher nur einzelne kleine schwarze Pertikelchen an dem Kerne haftend. Das dunkel glänzende Aussehen des Kerns verkündet aber schon, dass dieselbe das Pigment gelöst enthält und die Krystallisation erfolgen muss. Besonders charalkteristich für die Pigmentzellen ist ferner die gelbe Farbe des Kernkörperchens. Ich habe schon in dem früheren Aufsatze auf die Bedeutung des gelben Kernkörperchens für die Pigmentbildung hingewiesen und dieser Fall lässt dieselbe noch deutlicher dahin erkennen, dass wahrscheinlich das Kernkörperchen durch Mittheilung seines J'igmentes an den Kerninhalt den ersten Grund zu jener exosmotischen Strömung und zur Auskrystallisation des Zellenpigmentes auf der Aussenseite des Kernes bildet. Nach völliger Ausbildung der Pigmentzelle verlieren sowohl Kernkörperchen als auch der Kern ihre Farbe und ihren Glanz; die ausgebildete Pigmentzelle besitzt aber die Eigenschaft, Pigment zu produziren, nicht mehr, folglich müssen jene embryonalen 
Eigenschaften des Kernes und des Kernkörperchens dieser Production als ursächliche Momente zu Grunde liegen. - Weiter bestätigt sich aber meine frühere Behauptung, dass das Pigment und seine Production wesentlich die Bildung der äusseren Zelle veranlasst. In denjenigen Zellen, in welchen die Pigmentbildung noch nicht begonnen hatte, hob sich die Zellmembran nur als ein blasser Streifen von dem Kerne ab, lag also der Kernmembran oder dessen Begrenzung dicht an. Sobald sich aber auf dem Kerne schon einige Pigmenttheilchen abgelagert fanden, oder selbst einige Theilchen frei geworden waren, dehnte sich die Zellmembran stets nach irgend einer Seite aus und hob sich also weiter von dem Kerne ab. Von Pigmentbildung in den späteren Zellen des Chorioideastroma's konnte noch nicht die Rede sein, da die Chorioidea sich noch nicht von der Sclerotica und den Kopfplatten differenzirt hatte. Die Bildung dieser Zellen wird erst in der siebenten Woche ungefähr beginnen. Vermehrungsandeutungen labe ich in den Pigmentzellen niemals bemerkt, es bleibt daher nur übrig, anzunehmen, dass die Ausbildung der Epithellage durch Vergrösserung der Zellen vor sich geht.

Die zweite Form von Zellen war Jänglich (Fig. 5), die einfachste Form dieser Zellen fand sich nur sehr selten. Ein grosser Kern schien die Hauptmasse der Zelle völlig zu erfüllen und so weit war die Zelle dunkel und scharf contourirt (Fig. 5a). Dann dehnte sich aber in der gleichen Zellenbreite ein blasser Fortsatz nach einer Richtung hin (wahrscheinlich der inneren) aus. Innerhalb des Kernes lag ein kleines Kernkörperchen. Diese Zellen waren 0,004 Mm. breit, 0,015 Mm. lang; der Kern 0,01 Mm. Iang und 0,004 Mm. breit. Das dunkle Ende der Zelle war abgerundet, das blasse endete mit zwei rechtwinkligen Ecken. Wenn dies die einfachste Form dieser Zellen war, so stellten sich doch 
die meisten in einer von dieser sehr verschiedenen Form dar und bei der Geringfügigkeit des Materials liessen sich nicht alle Uebergangsstufen auffinden. Die grössere Nenge der Zellen stellte dunkelcontourirte Kegel dar. Die beiden langen Seiten convergirten in sehr spitzen Winkeln oder gingen abgerundet in einander über. Die dritte Seite stumpfte den Kegel gerade ab mit rechtwinkligen Ecken. Die Länge der Kegel betrug etwa 0,015 Mm., die durchschnittliche Breite 0,003 Mm. Die Seiten waren überall dunkel contourirt, nur an der Spitze fand sich regelmässig eine feine Unterbrechung der Contour. Von der Spitze des Kegels ging häufig ein höchst feiner Faden $a b$ und liess sich rückwärts durch jene Unterbrechung der Contour in das Innere der Zelle verfolgen. Er endigte nicht weit von dem entgegengesetzten Ende des Kegels mit einer kleinen Anschwellung. Aus der Beschreibung geht mit Sicherheit hervor, dass diese Zellenform die späteren Stäbchen bildet; es fand sich jedenfalls nur eine Form dieser Art, keine zweite, welche sich als Zapfen hätte bezeichnen lassen. Die Art und Weise, wie sich die Stäbchen aus jenen Zellen entwickeln, habe ich nicht genau verfolgen können. Die letzt beschriebene Form gleicht der bleibenden Stäbchenform bis auf die Grösse und das spitze innere Ende vollkommen. Aus der Membran der ursprünglichen Zelle geht die Hülle des Stäbchens hervor, aus dem Kerne scheint das Nark zu entstehen und das Kernkörperchen die Endigung des centralen Faden zu bilden. Wie der centrale Faden entsteht, kann ich leider nicht angeben. Den äusseren Faden kann man nach kurzem Verlaufe bis $z \mathbf{u}$ einer kleinen Anschwellung verfolgen und von dieser geht der Faden an der entgegengesetzten Seite weiter.

Die dritte Zellenform (Fig. 6) bestand aus runden Zellen von 0,0065 Mm. Durchmesser, welche vollständig von den runden $0,006 \mathrm{Mm}$. grossen Kernen erfüllt wur- 
den. Die Zellmembran lag dicht um den Kern und verstärkte seine Contour noch. Der Kern war nicht granulirt, glänzte ein wenig, nur selten befand sich in ihm ein kleines Kernkörperchen. Regelmässig hob sich die Zellmembran an zwei entgegengesetzten Polen von dem Kerne $\mathrm{ab}$ und bildete in beiden Richtungen feine, kaum doppelcontourirte Fortsätze. Im weitern Verlaufe enthielt der eine dieser Faden stets eine kleine Anschwellung, eine Ausdehnung des Fadens, welche zuweilen durch einen etwas dunkleren Inhalt markirt war. Offenbar entspricht diese Zellenform den Körnerzellen, deren Bedeutung ich in meiner Monographie*) über die Retina dargelegt habe, sie correspondiren ungefähr mit den inneren Körnern. Die kleinen Anschwellungen innerhalb der Faden, sowie innerhalb der früher beschriebenen Stäbchenfaden sind offenbar die ersten Andeutungen der (äusseren) Körner, welche keine Zellen sind und folglich auch keine selbstständige Entstehung haben können.

Die vierte Form der Zellen (Fig. 7) stimmt mit den Zellen des Hirns fast völlig überein. Sie haben einen Durchmesser von $0,008 \mathrm{Mm}$., ihre Membran hebt sich fast überall scharf von dem Kerne ab. Der Kern ist ebenfalls scharf begrenzt und enthält stets ein duukles Kernkörperchen. Von der Membran der Zelle gehen regelmässig mehrere doppelt contourirte Fortsätze ab; der Inhalt der Zelle ist höchst fein granulirt. Zuweilen findet sich an einer Zelle ein mit Varicositäten versehener Fortsatz. Alles dies beweist, dass die Zellenform den späteren Nervenzellen der Retina entspricht. Jene varicösen Fortsätze sind die ersten Andeutungen der Nervenfasern. Wir haben also vier Zellenformen gefunden, von denen drei zusammenhängend die Retina bilden.

*) Die Structur der Retina dargestellt nach Untersuchungen über das Auge des Wallfisches. Leipzig, bei W. Engelmann. 
Danach ist es gestattet anzunehmen, dass jedes Blatt der Augenblase gleichwerthig zwei Zellenformen und zwei Zellenreihen erzeugt, dass die Differencirung der Blätter in Zellen erst nach der Zusammenlagerung derselben beginnt und dann aus ursprünglich gleicher Anlage je eine Zellenform entsteht und zwar gleich mit der Anlage in völligem Zusammenhange mit den zugehörigen Theilen. Die Grösse der anfänglichen Kerne stimmt fast völlig überein, die Zellenbildung beginnt erst später und bedingt die Differencirung. Es sind dies eigentlich Postulate, welche man schon vor der Untersuchung aufstellen möchte. Den Stäbchen, Körnerzellell und Nervenzellen erweisen sich die (äusseren) Körmer nicht als gleichwerthig, sie entstehen erst später, wie jene Hauptbestandtheile der Retina, innerhalb der jene verbindenden Faden. Es liess sich dies aus der Function der Körner schon vermuthen, da diese erst mit dem eigentlichen Sehacte in Kraft tritt. Der Widerspruch mit.meiner früheren Arbeit in dieser Beziehung erklärt sich durch eine wesentlich andere Auffassung der beiden Körnerschichten, welche ich in der erwähnten Monographie entwickelt habe.

Nach dieser Betrachtung ergiebt sich ferner, dass die Retina im Beginne der fünften Woche derjenige Theil des Hiris ist, welcher am weitesten in der Entwickelung fortgeschritten ist. Die Form und der Zusammenhang der Zellen ist in der Retina am bestimmtesten ausgebildet und den späteren dauernden Verhältnissen am ähnlichsten. Ferner sind die der Retina besonders eigenthümlichen Theile, Stäbchen und Körnerzellen, ihrer bleibenden Gestalt am nächsten, sie würden nach meiner Annahme einer gleichen Lage entsprechen, der inneren Schicht der primären Augenblase. Ganglienzellen und Pigmentepithelien sind von der späteren Gestalt viel weiter entfernt. Was endlich die Vermehrung der Zellen 
anbetrifft, so habe ich fast gar keine Anhaltspunkte für eine solche gefunden. Es liess sich nur nachweisen, dass durch die Ausbildung der Zellen eine beträchtliche Vergrösserung der Membran eintreten muss, da dieselben ihrer späteren Grösse bei weitem nicht gleich kamen. Ferner nimmt die Retina in dieser Zeit den Raum einer Halbkugel ungefähr ein und wird erst durch die Bildung des Glaskörpers membranartig ausgedehnt. Allein diese beiden Punkte genügen zur Erklärung der nothwendigen Vergrösserung nur theilweise. Ich muss leider erklären, dass sowohl diese, wie die frühere Beobachtung mir zur Annahme einer Vermehrung der Zellenzahl auch nicht den geringsten Anhalt gegeben haben. 'Trotz grosser' Aufmerksamkeit habe ich von einer Theilung der Kerne niemals etwas bemerkt, wäre daher eher geneigt, eine völlige Neubildung von Kernen anzunehmen, falls sich nicht andere Wege zur Frklärung fänden. Veber die weitere Entwickelung der Zellen giebt die frühere Beobachtung einige Anhaltspunkte.

Juli 1864.

C. Ritter.

\section{Erklïrung der Figuren.}

Fig. 1. Zellen der Kopfplatten, aus denen Sclerotica und Chorioidea hervorgehen.

Fig. 2. Zellen der Linse.

Fig. 3. Zellen des Hirns.

Fig. 4. Zellen des Pigmentepithels der Chorioidea.

Fig. 5. Stäbchen nit centralen Faden.

a) Zellen, aus welcher die Stäbchen hervorgehen.

Fig. 6. Körnerzellen.

Fig. 7. Ganglienzellen.

Vergrösserung 290 . 